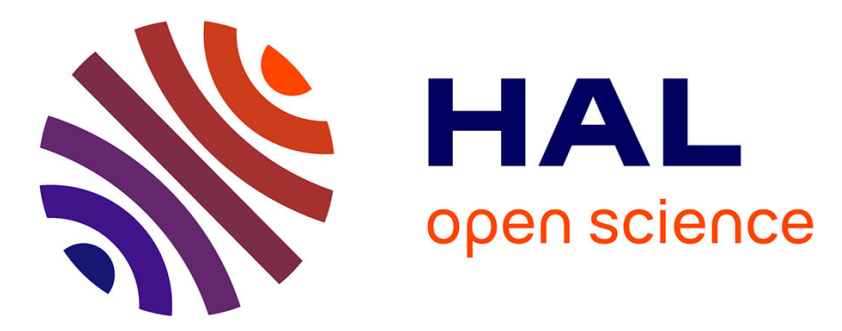

\title{
Heat and mass transfer analogies for evaporation models at high evaporation rate
}

P. Trontin, P. Villedieu

\section{To cite this version:}

P. Trontin, P. Villedieu. Heat and mass transfer analogies for evaporation models at high evaporation rate. AIAA AVIATION 2014, Jun 2014, ATLANTA, United States. hal-01069553

\section{HAL Id: hal-01069553 \\ https://hal-onera.archives-ouvertes.fr/hal-01069553}

Submitted on 29 Sep 2014

HAL is a multi-disciplinary open access archive for the deposit and dissemination of scientific research documents, whether they are published or not. The documents may come from teaching and research institutions in France or abroad, or from public or private research centers.
L'archive ouverte pluridisciplinaire HAL, est destinée au dépôt et à la diffusion de documents scientifiques de niveau recherche, publiés ou non, émanant des établissements d'enseignement et de recherche français ou étrangers, des laboratoires publics ou privés. 


\title{
Heat and mass transfer analogies for evaporation models at high evaporation rate.
}

\author{
P. Trontin and Ph. Villedieu \\ ONERA - The French Aerospace Lab, F-31055, Toulouse, France
}

\begin{abstract}
In the framework of anti and deicing applications, heated liquid films can appear above the ice thickness, or directly above the wall. Then, evaporation plays a major role in the Messinger balance and evaporated mass has to be predicted accurately. Unfortunately, it appears that existing models under-estimate evaporation at high temperature. In this study, different evaporation models at high evaporation rates are studied. The different hypothesis on which these models are built are discussed for high evaporation rates: ChiltonColburn analogy and non-zero blowing velocity at the film/air interface. These models are compared with a solution obtained by a finite volume approach where boundary layer equations are solved.
\end{abstract}

\section{Nomenclature}

$\dot{m}^{\prime \prime} \quad$ Surface mass flow rate $\left(k g \cdot m^{-2} \cdot s^{-1}\right)$

$\mathbf{u} \quad$ Velocity $\mathbf{u}=(u, v)$

Cf Friction coefficient

$D \quad$ Mass diffusivity

$h_{m} \quad$ Mass transfer coefficient

$h_{t} \quad$ Heat transfer coefficient

$L \quad$ Reference length

Le Lewis number $L e=\frac{S c}{P r}$

$L v \quad$ Latent heat of evaporation $L v=L v(T)$

$M \quad$ Mole mass

$P \quad$ Pressure

$\operatorname{Pr} \quad$ Prandtl number $\operatorname{Pr}=\frac{\nu}{\alpha}$

Psat Saturation vapor pressure Psat $=P$ sat $(T)$

$r_{H} \quad$ Relative humidity of air

Re Reynolds number

$S c \quad$ Schmidt number $S c=\frac{\nu}{D_{v}}$

$S t_{m} \quad$ Mass Stanton number

$S t_{t} \quad$ Thermal Stanton number

$T$ Temperature

$X \quad$ Mole fraction

$Y \quad$ Mass fraction

\section{Subscripts}

$0 \quad$ Liquid film/gas boundary layer interface (corresponds to $y=0$ )

$\infty \quad$ Outside limit of the gas boundary layer (corresponds to $y=\delta$ )

a Relative to dry air

$g \quad$ Relative to gas (dry air+steam)

$l \quad$ Relative to liquid water (inside the liquid film)

$v \quad$ Relative to steam (water vapor)

Symbols 


$\begin{array}{ll}\alpha & \text { Thermal diffusivity } \\ \delta & \text { Thickness of the dynamic boundary layer for the gas } \\ \delta_{M} & \text { Thickness of the mass boundary layer for the gas } \\ \delta_{T} & \text { Thickness of the thermal boundary layer for the gas } \\ \mu & \text { Dynamic viscosity } \\ \rho & \text { Density }\end{array}$

Superscripts

$0 \quad$ Low evaporation rate (blowing velocity at the film/air interface is supposed to be $0\left(v_{g 0}=0\right)$ )

\section{Introduction}

Ice growth prediction on aircraft is a fundamental problem for aeronautical applications. Ice accretion can indeed modify local shapes of wings or blades and cause aerodynamic performance losses. Ice shedding can clog air intakes too. Models dedicated to ice prediction are all based on the Messinger approach. ${ }^{1}$ This model is mainly based on both mass and energy balances between ice thickness itself, incoming droplets, extern atmosphere (through evaporation and heat transfer) and runback film.

Ice protection systems aim at keeping ice accretion under control. To do this, heat from the engine or from electrical resistor can be used. In that case, a heated liquid film can appear above the ice thickness, or directly above the wall. A challenging point is to calibrate ice protection system and the amount of heat to be extracted to prevent runback film from re-freezing downstream. In particular, evaporation rate $\dot{m}_{v}^{\prime \prime}$ has to be estimated accurately. Indeed, in most icing tools like ONICE for example, evaporation rate tends to be under-estimated, which leads ice protection systems to be too conservative. Classical evaporation models used in icing codes like ONICE, LEWICE or CANICE behave like the product between a mass transfer coefficient $h_{m}^{0}$ and the gap $Y_{v 0}-Y_{v \infty}$ :

$$
\dot{m}_{v}^{\prime \prime} \approx \rho \cdot h_{m}^{0} \cdot\left(Y_{v 0}-Y_{v \infty}\right)
$$

Therefore, the computation of $\dot{m}_{v}^{\prime \prime}$ is focused on the one of $h_{m}^{0}$. Mass transfer coefficient appears to be a mean coefficient related to evaporation from a global point of view, without solving the boundary layer itself.

The aim of this paper is to study behaviors of different evaporation models for high evaporation rates. The different hypothesis on which these models are built are discussed for high evaporation rates: ChiltonColburn analogy ${ }^{2}$ and non-zero blowing velocity at the film/air interface. These models will be compared with a solution obtained by a finite volume approach where boundary layer equations are solved.

In a first part, the boundary layer equations are presented. Definition of the evaporation rate $\dot{m}_{v}^{\prime \prime}$ is given too. In a second part, the Reynolds and Chilton-Colburn analogies, used to define $h_{m}$ respectively from $C f$ and $h_{t}$, are presented. Limits of analogies for high evaporation rates are emphasized. In a third part, different models for $\dot{m}_{v}^{\prime \prime}$ are presented. Evaporation rates are computed and compared with the solution obtained by a finite volume approach where boundary layer equations are solved. Test case is a heated plane plate and accuracy of the models are measured for different evaporation rates (from $T_{0}=0^{\circ} C$ to $T_{0} \approx 100^{\circ} C$ ). Finally, conclusions are drawn.

\section{Equations and evaporation rate}

\section{II.A. Configuration}

Configuration is shown in Fig. 1. A water liquid film runs back over a plane plate. An incompressible, laminar air boundary layer develops above the liquid film (Fig. 1). Liquid film is supposed to be locally plane so that surface tension forces can be neglected. Liquid film/air interface is located at $y=0$ and the boundary layer develops from $y=0$ to $y=\delta$. Connexion with the inviscid flow at $y=\delta$ is such as $\mathbf{u}_{\mathbf{g}}(x, \delta)=U_{\infty} \mathbf{e}_{\mathbf{x}}$

\section{II.B. Equations}

Concerning the incompressible laminar boundary layer, following hypothesis are made:

- Gas flow is supposed to be stationary 
- Flow outside the boundary layer $y>\delta$ does not depend on $x$. Therefore, $U_{g \infty}(x)=U_{\infty}$ and so the pressure gradient $\frac{d P}{d x}$ is zero.

- Physical properties as $\left(\rho_{g}, \mu_{g}, \alpha_{g}\right.$ et $\left.D_{v}\right)$ are supposed to remain constant inside the boundary layer

Dimensionless boundary layer equations are given by:

$$
\begin{array}{r}
\frac{\partial \tilde{u}_{g}}{\partial \tilde{x}}+\frac{\partial \tilde{v}_{g}}{\partial \tilde{y}}=0 \\
\tilde{u}_{g} \frac{\partial \tilde{u}_{g}}{\partial \tilde{x}}+\tilde{v}_{g} \frac{\partial \tilde{u}_{g}}{\partial \tilde{y}}-\frac{1}{R e} \frac{\partial^{2} \tilde{u}_{g}}{\partial \tilde{y}^{2}}=0 \\
\tilde{u}_{g} \frac{\partial \tilde{T}_{g}}{\partial \tilde{x}}+\tilde{v}_{g} \frac{\partial \tilde{T}_{g}}{\partial \tilde{y}}-\frac{1}{R e \cdot \operatorname{Pr}} \frac{\partial^{2} \tilde{T}_{g}}{\partial \tilde{y}^{2}}=0 \\
\tilde{u}_{g} \frac{\partial \tilde{Y}_{v}}{\partial \tilde{x}}+\tilde{v}_{g} \frac{\partial \tilde{Y}_{v}}{\partial \tilde{y}}-\frac{1}{R e \cdot S c} \frac{\partial^{2} \tilde{Y}_{v}}{\partial \tilde{y}^{2}}=0
\end{array}
$$

where the dimensionless variables are given by:

$$
\tilde{x}=\frac{x}{L} \quad ; \quad \tilde{y}=\frac{y}{L} \quad ; \quad \tilde{u}_{g}=\frac{u_{g}}{U_{\infty}} \quad ; \quad \tilde{v}_{g}=\frac{v_{g}}{U_{\infty}} \quad ; \quad \tilde{T}_{g}=\frac{T_{g}-T_{g 0}}{T_{g \infty}-T_{g 0}} \quad ; \quad \tilde{Y}_{v}=\frac{Y_{v}-Y_{v 0}}{Y_{v \infty}-Y_{v 0}}
$$

Boundary conditions are the following ones:

- At $y=0$ :

$$
\tilde{u}_{g}(y=0)=\tilde{u}_{g 0}=0 \quad ; \quad \tilde{T}_{g}(y=0)=\tilde{T}_{g 0}=0 \quad ; \quad \tilde{Y}_{v}(y=0)=\tilde{Y}_{v 0}=0
$$

- At $y=\delta$ :

$$
\tilde{u}_{g}(y=\delta)=1 \quad ; \quad \tilde{T}_{g}(y=\delta)=1 \quad ; \quad \tilde{Y}_{v}(y=\delta)=1
$$

In the rest of the manuscript, the ${ }^{\sim}$ notation is dropped.

Mass fraction $Y_{v 0}$ and $Y_{v \infty}$ can be written:

$$
Y_{v 0}=\frac{X_{v 0}}{X_{v 0}+\frac{M_{a}}{M_{v}}\left(1-X_{v 0}\right)} \quad ; \quad Y_{v \infty}=\frac{X_{v \infty}}{X_{v \infty}+\frac{M_{a}}{M_{v}}\left(1-X_{v \infty}\right)}
$$

Thermodynamic equilibrium is assumed at the liquid film inteface so that $P_{v 0}=\operatorname{Psat}_{v}\left(T_{g 0}\right)$. Saturation vapor pressure $P_{v 0}=\operatorname{Psat}_{v}\left(T_{g 0}\right)$ can be computed using of Clausius-Clapeyron law for example. Therefore $X_{v 0}=\frac{P_{v 0}}{P_{g 0}}=\frac{P_{s a t}\left(T_{g 0}\right)}{P_{g 0}}$ and it appears that Eq. (2)(d) is coupled to thermal equation (2)(c) via the boundary condition on $Y_{v 0}$.

Outside the boundary layer, $X_{v \infty}=\frac{P_{v \infty}}{P_{g \infty}}=r_{H} \cdot \frac{P s a t_{v}\left(T_{g \infty}\right)}{P_{g \infty}}$.

$U_{\infty}, T_{g 0}, T_{g \infty}$ are known and set by either outside flow or film properties.

\section{II.C. Evaporation rate at the film/air interface $(y=0)$}

Evaporation rate at the film/air interface derives from the vapor flux through the interface. Mass flux conservation through the film/air interface can be written:

$$
\rho_{g 0}\left(v_{g 0}-v_{I}\right)=\rho_{l 0}\left(v_{l 0}-v_{I}\right)=\dot{m}_{v}^{\prime \prime}
$$

where $v_{I}$ is the film/air interface velocity. Liquid film is supposed to be at rest and $v_{l 0}=0$. Therefore, $\left|v_{I}\right|=\left|v_{I}-v_{l 0}\right|=\frac{\rho_{g 0}}{\rho_{l 0}}\left|v_{I}-v_{g 0}\right|$. In the case of of a water/air interface, $\rho_{g} \approx 1 \mathrm{~kg} \cdot \mathrm{m}^{-3}$ and $\rho_{l} \approx 1000 \mathrm{~kg} \cdot \mathrm{m}^{-3}$ which gives $\frac{\left|v_{I}-v_{g 0}\right|}{\left|v_{I}\right|}>>1$. Then, $\left|v_{g 0}\right|>>\left|v_{I}\right|$ and $v_{g 0}-v_{I} \approx v_{g 0}$. Finally $\dot{m}_{v}^{\prime \prime}=\rho_{g 0} v_{g 0}$.

Dry air does not dissolve inside water through interface ${ }^{3}$ so that $\rho_{a 0} v_{a 0}=0$ and $\dot{m}_{v}^{\prime \prime}=\rho_{g 0} v_{g 0}=\rho_{a 0} v_{a 0}+$ $\rho_{v 0} v_{v 0}=\rho_{v 0} v_{v 0}$. The vapor flux can be splitted into contributions of convective flux and diffusive flux (Fick 
law): $\rho_{v 0} v_{v 0}=Y_{v 0} \rho_{g 0} v_{g 0}-\left.\rho_{g 0} D_{v 0} \frac{\partial Y_{v}}{\partial y}\right|_{0}$. Evaporation rate can be written: $\dot{m}_{v}^{\prime \prime}=Y_{v 0} \rho_{g 0} v_{g 0}-\left.\rho_{g 0} D_{v 0} \frac{\partial Y_{v}}{\partial y}\right|_{0}=$ $Y_{v 0} \dot{m}_{v}^{\prime \prime}-\left.\rho_{g 0} D_{v 0} \frac{\partial Y_{v}}{\partial y}\right|_{0}$. Finally:

$$
\dot{m}_{v}^{\prime \prime}=-\left.\frac{D_{v} \cdot \rho_{0}}{1-Y_{v 0}} \frac{\partial Y_{v}}{\partial y}\right|_{y=0}
$$

Figure 2 represents $Y_{v 0}$ vs. $T_{g 0}$. For classical icing applications (no anti- or de-icing), $T_{g 0}$ is between $-20^{\circ} C$ and $O^{\circ} C$ so that $Y_{v 0}$ is between 0.001 and 0.005 . For anti- or de-icing configurations, plate is heated and liquid film temperature can be greater than $80^{\circ} \mathrm{C}$. In this case, $Y_{v 0} \longrightarrow 1$ and Eq. (3) becomes singular with very large mass flow rate.

\section{Computation of $\dot{m}_{v}^{\prime \prime}$. Presentation of Reynolds and Chilton-Colburn analogies. Limits for high evaporation rates}

As can be seen in Eq. (3), evaporated mass $\dot{m}_{v}^{\prime \prime}$ is obtained by the computation of the gradient $\left.\frac{\partial Y_{v}}{\partial y}\right|_{y=0}$. Several approaches are possible:

- Evaporated mass can be computed indirectly by using a mass transfer coefficient $h_{m}$ (which depends itself on the gradient of $Y_{v}$ at the film/air interface). $h_{m}$ is obtained from the friction coefficient $C f$ or the heat ransfer coefficient $h_{t}$ via a Reynolds or a Chilton-Colburn analogy. ${ }^{3,4}$ This analogies are exact in the context of a laminar boundary layer without pressure gradient (Blasius solution) on a plane liquid film. This will be presented in this part as well as the validity of the analogies outside this context.

- Evaporated mass can be obtained directly by computing the gradient of $Y_{v}$ at the film/air interface. This can be done for example by a boundary layer code.

\section{III.A. Definition of $\dot{m}_{v}^{\prime \prime}$ in function of $h_{m}$}

First, some definitions are given. Coefficients (function of $\left.\frac{\partial Y_{v}}{\partial y}\right|_{y=0}$ ) are introduced, notably the mass transfer coefficient $h_{m}$. Evaporation rate $\dot{m}_{v}^{\prime \prime}$ is written in function of $h_{m}$, which focus all the difficulty of the computation of $\dot{m}_{v}^{\prime \prime}$ into $h_{m}$.

\section{III.A.1. Some definitions}

Friction coefficient $C f$, heat transfer coefficient $h_{t}$ and mass transfer coefficient $h_{m}$ are defined as:

$$
\begin{gathered}
C f=\frac{\left.\mu_{g} \frac{\partial u_{g}}{\partial y}\right|_{y=0}}{\frac{1}{2} \rho_{g e} u_{g e}^{2}} \\
h_{t}=\frac{\left.\lambda_{g} \frac{\partial T_{g}}{\partial y}\right|_{y=0}}{T_{g e}-T_{g 0}} \\
h_{m}=\frac{\left.D_{v} \frac{\partial Y_{v}}{\partial y}\right|_{y=0}}{Y_{v e}-Y_{v 0}}
\end{gathered}
$$

Dimensionless formulation for $h_{t}$ and $h_{m}$ can be obtained by using Nusselt $N u$ and Sherwood numbers $S h$ :

$$
\begin{aligned}
N u & =\frac{h_{t} L}{\lambda_{g}} \\
S h & =\frac{h_{m} L}{D_{v}}
\end{aligned}
$$


Thermal $S t_{t}$ and mass $S t_{m}$ Stanton numbers can be written:

$$
\begin{aligned}
S t_{t} & =\frac{N u}{P r \cdot R e} \\
S t_{m} & =\frac{S h}{S c \cdot R e}
\end{aligned}
$$

Skin friction $\tau$, diffusive heat flux $\Phi_{t}^{\text {diff }}$ and diffusive mass flux $\Phi_{m}^{\text {diff }}$ are given by:

$$
\begin{aligned}
\tau & =\frac{1}{2} C f \cdot \rho_{\infty} \cdot U_{\infty}^{2} \\
\Phi_{t}^{d i f f} & =S t_{t} \cdot \rho_{\infty} \cdot U_{\infty} \cdot C p \cdot\left(T_{g \infty}-T_{g 0}\right) \\
\Phi_{m}^{d i f f} & =S t_{m} \cdot \rho_{\infty} \cdot U_{\infty} \cdot\left(Y_{v \infty}-Y_{v 0}\right)
\end{aligned}
$$

III.A.2. Evaporated mass $\dot{m}_{v}^{\prime \prime}$ in function of $h_{m}$

Evaporated mass $\dot{m}_{v}^{\prime \prime}$ (Eq. (3)) is written by using $h_{m}$ coefficient (Eq. (4)):

$$
\dot{m}_{v}^{\prime \prime}=-\rho_{g 0} h_{m} \frac{Y_{v \infty}-Y_{v 0}}{1-Y_{v 0}}
$$

Computation of $\dot{m}_{v}^{\prime \prime}$ consists now in the one of $h_{m}$. If mass fraction $Y_{v}$ is written as: $\rho_{g} Y_{v}=\frac{M_{v}}{R} \frac{P_{v}}{T_{g}}$, one has finally:

$$
\dot{m}_{v}^{\prime \prime}=\frac{-h_{m}}{1-Y_{v 0}} \frac{M_{v}}{R}\left(\frac{r_{H} \cdot P_{s a t_{v}}\left(T_{g \infty}\right)}{T_{g \infty}}-\frac{\text { Psat }_{v 0}\left(T_{g 0}\right)}{T_{g 0}}\right)
$$

which is strictly equivalent to Eq. (7).

In many codes (ONICE or LEWICE for example), Eq. (8) is used to compute evaporation rate but with following approximations:

- $\frac{1}{1-Y_{v 0}} \approx 1$ which corresponds to low evaporation rates (see Fig. 2)

- $h_{m}=h_{m}^{0}$, i.e. mass transfer coefficient is computed without taking into account blowing at the film/air interface due to evaporation velocity

\section{III.B. Reynolds and Chilton-Colburn analogies for the computation of $h_{m}$}

Analogies beween $C f, h_{t}$ (or $\left.S t_{t}\right)$ and $h_{m}$ (or $S t_{m}$ ) are established in this section. These analogies can be derived rigorously in the context of a flate laminar boundary layer with no pressure gradient. Such a configuration has been retained in this study (see section II). Equation (2) can be re-written by using the self-similar properties of the solution: ${ }^{5}$

$$
\left\{\begin{array}{l}
u_{g}=F^{\prime}(\eta) \\
v_{g}=\frac{1}{2} \frac{y}{x} F^{\prime}(\eta)-\frac{1}{2} \sqrt{\frac{\nu}{U_{\infty} x}} F(\eta)
\end{array}\right.
$$

where $F$ is a function of the only variable $\eta=y \sqrt{\frac{U_{\infty}}{\nu x}}$. Functions $\theta(\eta)$ and $\Phi(\eta)$ are defined likewise: $\theta(\eta)=T_{g}(x, y)$ and $\Phi(\eta)=Y_{v}(x, y)$. Finally, Eq. (2) can be written:

$$
\begin{aligned}
F^{\prime \prime \prime}+\frac{1}{2} F \cdot F^{\prime \prime} & =0 \\
\theta^{\prime \prime}+\frac{1}{2} \operatorname{Pr} \cdot F \cdot \theta^{\prime} & =0 \\
\Phi^{\prime \prime}+\frac{1}{2} S c \cdot F \cdot \Phi^{\prime} & =0
\end{aligned}
$$

Important remark : no hypothesis has been done yet about the shape of $v_{g 0}$.

In the rest of the document, developments will be performed for temperature (Eq. (10)(b)). Conclusions for vapor mass fraction $Y_{v}$ (Eq. (10)(c)) are similar. 
III.B.1. Case $\operatorname{Pr}=S c=1$

Here the three equations of Eq. (10) are similar. Therfore $F^{\prime \prime}=\theta^{\prime}=\Phi^{\prime}$ and particularly $F^{\prime \prime}(0)=\theta^{\prime}(0)=$ $\Phi^{\prime}(0)$. Then, $\left.\frac{1}{U_{\infty}} \frac{\partial u_{g}}{\partial y}\right|_{0}=\left.\frac{1}{T_{\infty}-T_{g 0}} \frac{\partial T_{g}}{\partial y}\right|_{0}=\left.\frac{1}{Y_{v \infty}-Y_{v 0}} \frac{\partial Y_{v}}{\partial y}\right|_{0}$. Finally for $\operatorname{Pr}=S c=1$ :

$$
\frac{C f}{2}=S t_{t}=S t_{m}
$$

It is possible to link $S t_{m}$ (and so $h_{m}$ ) with the friction coefficient $C f$. The interesting feature is that no hypothesis has been done about $v_{g 0}$ so that Eq. (11) is valid even for large evaporation rates. But for air, $\operatorname{Pr} \approx 0.7$ and $S c \approx 0.6$.

\section{III.B.2. Case $\operatorname{Pr} \neq 1$ and $S c \neq 1$}

Hypothesis: blowing velocity at the film/air interface due to evaporation is set to zero $\left(v_{g 0}=0\right)$. Thus, from Eq. (9): $F^{\prime}(0)=0$ and $F(0)=0$. From Eq. (10)(a), we have $F^{\prime \prime \prime}(0)=0$, and then $F^{(4)}(0)=0$. Near $\eta=0(y=0)$ :

$$
F(\eta) \underset{\eta \rightarrow 0}{=} F^{\prime \prime}(0) \frac{\eta^{2}}{2}+O\left(\eta^{5}\right)
$$

Integration of Eq. (10)(b) gives: $\theta^{\prime}(\eta)=\theta^{\prime}(0) \exp \left[-\frac{1}{2} \int_{0}^{\eta} \operatorname{Pr} \cdot F(\xi) d \xi\right]$. Let $a(\eta, P r)$ be $a(\eta, P r)=$ $\exp \left[-\frac{1}{2} \int_{0}^{\eta} \operatorname{Pr} \cdot F(\xi) d \xi\right]$. Integrating previous equation one more time and given that $\theta(0)=0$, we get : $\theta(\eta)=\theta^{\prime}(0) \int_{0}^{\eta} a(\xi, P r) d \xi$. As $\theta(\infty)=1$, finally:

$$
\theta^{\prime}(0)=\frac{1}{\int_{0}^{\infty} a(\xi, \operatorname{Pr}) d \xi}
$$

Introducing Eq. (12) in the definition of $a$ :

$$
a(\eta, P r)=\exp \left[-\frac{1}{2} \int_{0}^{\eta} \operatorname{Pr} \cdot\left(F^{\prime \prime}(0) \frac{\xi^{2}}{2}+O\left(\xi^{5}\right)\right) d \xi\right]
$$

Previous expression is the more accurate as $\operatorname{Pr}$ number is large. Let now suppose that $\operatorname{Pr}>>1$ :

$$
a(\eta, P r) \approx \exp \left[-\frac{1}{12} \operatorname{Pr} \cdot F^{\prime \prime}(0) \eta^{3}\right]
$$

and then:

$$
\theta^{\prime}(0) \approx \frac{1}{\int_{0}^{\infty} \exp \left[-\frac{1}{12} \operatorname{Pr} \cdot F^{\prime \prime}(0) \xi^{3}\right] d \xi}
$$

The following change of variables $\zeta=P r^{1 / 3} \xi$ is performed:

$$
\begin{aligned}
& \theta^{\prime}(0) \approx \frac{\operatorname{Pr}^{1 / 3}}{\int_{0}^{\infty} \exp \left[-\frac{1}{12} \cdot F^{\prime \prime}(0) \zeta^{3}\right] d \zeta} \\
& \theta^{\prime}(0) \approx \frac{P r^{1 / 3}}{\int_{0}^{\infty} a(\zeta, \operatorname{Pr}=1) d \zeta} \\
& \theta^{\prime}(0) \approx \operatorname{Pr}^{1 / 3} \cdot F^{\prime \prime}(0)
\end{aligned}
$$

A strictly equivalent argument for $\Phi$ and $S c$ leads to: $\Phi^{\prime}(0) \approx S c^{1 / 3} \cdot F^{\prime \prime}(0)$ for $S c>>1$. Finally with dimensionless coefficients:

$$
\begin{aligned}
S t_{t}^{0} & =\frac{C f^{0}}{2} P r^{-2 / 3} \\
S t_{m}^{0} & =\frac{C f^{0}}{2} S c^{-2 / 3}
\end{aligned}
$$

These are Reynolds analogies. Ratio between $S t_{t}$ et $S t_{m}$ allows to write:

$$
\frac{h_{m}^{0}}{h_{t}^{0}}=\frac{L e^{-2 / 3}}{\rho_{g} \cdot C p}
$$


which is called the Chilton-Colburn analogy. Superscript ${ }^{0}$ is here to keep in mind that the Chilton-Colburn analogy and the Reynolds analogy have been obtained with $v_{g 0}=0$ which corresponds to low evaporation rate.

III.B.3. Prandtl and Schmidt number influence in the Reynolds analogy

We propose to study the influence of Prandtl $\operatorname{Pr}$ and Schmidt $S c$ numbers on the Reynolds analogy (Eq. (13)). Evaporation rate is neglected in this study. Blasius theory provides: ${ }^{5} \frac{C f}{2} \approx 0.332 R e_{x}^{-1 / 2}$ where $R e_{x}=\frac{U_{\infty} x}{\nu_{\infty}}$. Using Reynolds analogies (Eq. (13)), one has:

$$
\begin{aligned}
& S t \cdot \sqrt{R e_{x}} \cdot \operatorname{Pr}^{2 / 3}=c s t \approx 0.332 \\
& S h \cdot \sqrt{R e_{x}} \cdot S c^{2 / 3}=c s t \approx 0.332
\end{aligned}
$$

In Tab. 1, numerical results are shown. They are obtained by a finite volume approach where boundary layer equations are solved. The dimensionless $S t \cdot \sqrt{R e_{x}} \cdot \mathrm{Pr}^{2 / 3}$ is computed and results are compared with those deriving from a Reynolds analogy (Eq. (15)). Different $\operatorname{Pr}$ numbers are studied. Similar conclusions for $S c$ numbers can be drawn. In this computation, $U_{\infty}=100 \mathrm{~m} . \mathrm{s}^{-1}$. Well known results about $\operatorname{Pr}$ influence on Reynolds analogy are shown in Tab. 1: the analogy is accurate for high Prandtl number. For air, $\operatorname{Pr} \approx 0.7$ and the error for the Reynolds analogy is around $2 \%$.

\section{III.C. Spalding approach for $\dot{m}_{v}^{\prime \prime}$ computation}

In this section, the approach proposed by Spalding ${ }^{4}$ is proposed. Mass conservation for steam can be written:

$$
\nabla \cdot\left(\rho_{v} \mathbf{u}_{v}\right)=\frac{\partial\left(\rho_{v} u_{v}\right)}{\partial x}+\frac{\partial\left(\rho_{v} v_{v}\right)}{\partial y}=0
$$

Mass conservation for gaz (dry air+steam) is:

$$
\nabla \cdot\left(\rho_{g} \mathbf{u}_{g}\right)=\frac{\partial\left(\rho_{g} u_{g}\right)}{\partial x}+\frac{\partial\left(\rho_{g} v_{g}\right)}{\partial y}=0
$$

The major hypothesis is that the flow is supposed to remain the same in the $\mathbf{e}_{x}$ direction so that $\frac{\partial}{\partial x}=0$. Therfore:

$$
\begin{array}{ll}
\rho_{v} v_{v}=\rho_{v 0} v_{v 0}=\dot{m}_{v}^{\prime \prime} & \forall y \\
\rho_{g} v_{g}=\rho_{g 0} v_{g 0}=\dot{m}_{v}^{\prime \prime} & \forall y
\end{array}
$$

Evaporation rate can be written: $\dot{m}_{v}^{\prime \prime}=\rho_{v} v_{v}=Y_{v} \rho_{g} v_{g}-\rho_{g} D_{v} \frac{\partial Y_{v}}{\partial y}=Y_{v} \dot{m}_{v}^{\prime \prime}-\rho_{g} D_{v} \frac{d Y_{v}}{d y}$. Then, $\frac{d Y_{v}}{Y_{v}-1}=\frac{\dot{m}_{v}^{\prime \prime}}{\rho_{g} D_{v}} d y$. The last equation is integrated from the film/air interface $(y=0)$ and $\delta_{M}$ where $Y_{v}$ is supposed to be $Y_{v \infty}$. Finally:

$$
\dot{m}_{v}^{\prime \prime}=\frac{\rho_{g 0} D_{v}}{\delta_{M}} \ln (1+B)
$$

where $B$ is defined by:

$$
B=\frac{Y_{v \infty}-Y_{v 0}}{Y_{v 0}-1}
$$

As $\delta_{M}$ thickness is unknown, $\delta_{M}$ is expressed in function of $h_{m}$. Using Eq. (7), (16) and (17), one gets:

$$
\frac{D_{v}}{\delta_{M}} \frac{\ln (1+B)}{B}=h_{m}
$$

For low evaporation rates, $B \rightarrow 0$ and $\frac{D_{v}}{\delta_{M}^{0}} \underset{B \rightarrow 0}{\approx} h_{m}^{0}$. Therefore, $h_{m}=h_{m}^{0} \frac{\ln (1+B)}{B} \frac{\delta_{M}^{0}}{\delta_{M}}$ and finally:

$$
\dot{m}_{v}^{\prime \prime}=\rho_{g 0} \cdot h_{m}^{0} \cdot \frac{\ln (1+B)}{B} \cdot B \cdot \frac{\delta_{M}^{0}}{\delta_{M}}
$$

If $h_{m}^{0}$ is computed from $h_{t}^{0}$ with a Chilton-Colburn analogy (Eq. (14)):

$$
\dot{m}_{v}^{\prime \prime}=\frac{h_{t}^{0}}{C p_{a} \cdot L e^{2 / 3}} \ln (1+B) \frac{\delta_{M}^{0}}{\delta_{M}}
$$


Equation (18) has been derived with the hypothesis that $\frac{\partial}{\partial x}=0$ which is not valid for a boundary layer. Indeed, the boundary layer thickness behaves like $\sqrt{R e_{x}}$ and the approximation $\frac{\partial}{\partial x}=0$ may have sense far from the stagnation point, but is strongly wrong near the stagnation point where the boundary layer is initiated. Unfortunately, for anti or de-icing applications, heated zones are located near the leading edge where the approximation $\frac{\partial}{\partial x}=0$ is not valid for the boundary layer.

\section{Comparison of different evaporation models}

Different models are compared with the reference case which corresponds to a finite volume approach where boundary layer equations are solved. Evaporation rates $\dot{m}_{v}^{\prime \prime}$ will be compared. A wide range of evaporation rates are studied, from $T_{0}=0^{\circ} \mathrm{C}$ to $T_{0} \approx 100^{\circ} \mathrm{C}$.

\section{IV.A. Presentation of the different models for $\dot{m}_{v}^{\prime \prime}$}

- Model (1): Results derive from a boundary layer code where the equations (2)(a), (2)(b), (2)(c) and (2)(d) are solved numerically by a finite volume method. These equations are coupled so that the blowing velocity at the film/air interface which is a boundary condition for Eq. (2)(b) is obtained from evaporation velocity deriving from Eq. (2)(d). A grid convergence has been performed so that the solution obtained with this model can be considered to be exact. It will be the reference solution.

- Model (2): This model is based on two approximations. First, analogy $S t_{m}^{0}=\frac{C f^{0}}{2} S c^{-2 / 3}$ (Eq. (13)) is used as well as the exact relation between $h_{m}^{0}$ and $S t_{m}^{0}$. Secondly, $C f^{0}$ is given by the classical expression on a laminar plane plate $\left(C f^{0} \approx 0.664 \cdot R e_{x}^{-1 / 2}\right)$. Blowing due to evaporation is not taken into account to compute $h_{m}^{0}$. Finally, $\dot{m}_{v}^{\prime \prime}$ is computed with Eq. (7) which is valid even for large evaporation rates.

- Model (3): $\dot{m}_{v}^{\prime \prime}$ computed by model (2) is multiplied by the Spalding correction $\frac{\ln (1+B)}{B}$ to obtain the evaporation rate given by Eq. (18)

- Model (4): This model is similar to model (2) but with one less approximation. Here, exact $C f$ is used (deriving from the exact numerical solution with a blowing velocity due to evaporation at the film/air interface). Finally, $\dot{m}_{v}^{\prime \prime}$ is computed with Eq. (7).

- Model (5): This model is similar to model (4) except that direct analogy between $h_{t}$ and $h_{m}$ (ChiltonColburn analogy Eq. (14)) is used. $h_{t}$ coefficient is obtained from the heat flux numerically computed at the film/air interface with a blowing velocity due to evaporation. Note that the Chilton-Colburn analogy (Eq. (14)) has been initially derived for low evaporation rate. In this model, it is used even for large evaporation rates.

- Model (6): This model is the one classically used in the ONERA code ONICE (and in almost all icing tools in the world like NASE LEWICE code) for low evaporation rates, i.e. $\dot{m}_{v}^{\prime \prime}=\rho_{g 0} h_{m}^{0}\left(Y_{v 0}-Y_{v e}\right)$. Coefficient $h_{m}^{0}$ is computed as done in ONICE, i.e. like in the model (2). No specific correction is taken into account to deal with large evaporation rate and deriving blowing velocities at the film/air interface.

\section{IV.B. Numerical study}

The context of the study is a liquid film on a plane plate. Above the film, a laminar gas boundary layer is developed. Pressure gradient is supposed to be zero. Incompressible equations are treated. The film is heated so that a study on $T_{g 0}$ is performed. Parameters are:

$$
\left\{\begin{array}{l}
U_{\infty}=100 \mathrm{~m} \cdot \mathrm{s}^{-1} \\
P_{\infty}=1013.25 \mathrm{hPa} \\
T_{\infty}=-5^{\circ} \mathrm{C} \\
r_{H}=1.0
\end{array}\right.
$$

Results are represented in Tab. 2 and 3. To take into accound self-similar properties of the boundary layer, $\dot{m}_{v}^{\prime \prime} \cdot R e_{x}$ is represented. 
For low evaporation rates $\left(T_{g 0}=0^{\circ} \mathrm{C}\right.$ and $\left.20^{\circ} \mathrm{C}\right)$ all the models are accurate. This is because all the models degenerate to a single model for low evaporation rate (when $Y_{v 0} \rightarrow Y_{v \infty}$ ). Conclusions are different for high evaporation rates $\left(T_{g 0}=85^{\circ} \mathrm{C}\right.$ et $\left.99.9^{\circ} \mathrm{C}\right)$. Models (2) and (3) give bad results. Indeed, the friction coefficient $C f^{0}$ used to compute $h_{m}$ does not take into account the blowing velocity at film/air interface due to evaporation. To understand major importance of such velocity on friction coefficient, Fig. 3 shows velocity profiles in the boundary layer when film temperature is maintained at $0^{\circ} \mathrm{C}$ (left) and $99.9^{\circ} \mathrm{C}$ (right). First, we can notice that the boundary layer thickness at high evaporation rates is larger than at low evaporation rates. Secondly, the gradient $\left.\frac{\partial u}{\partial y}\right|_{0}$ at the film/air interface (directly linked to the computation of $C f$ ) is widely larger at low evaporation than at high evaporation rates. Then, with model (2) where blowing velocity due to evaporation is not taken into account, $C f$ is widely over-estimated and resulting $h_{m}$ and $\dot{m}_{v}^{\prime \prime}$ are not accurate. Model (4) takes blowing velocity into account and is more accurate. Globally, model (5) gives the best results (error less than $5 \%$ up to $85^{\circ} \mathrm{C}$ ), but $h_{t}$ is supposed to be known at the film/air interface with a blowing velocity, which is not the case in the great majority of codes available in the icing community.

It is surprising and interesting to notice that model (6), which corresponds to the model used in the great majority of icing codes, gives accurate results both for low and high evaporation rates. Such a good accuracy seems to result from a compensation between three errors:

- $C f$ and $h_{t}$ are computed without taking into account blowing velocity at the film/air interface due to evaporation. Therefore $C f$ and $h_{t}$ are over-estimated.

- Chilton-Colburn analogies are not exact (especially for high evaporation rates)

- Evaporation rate is computed by:

$$
\dot{m}_{v}^{\prime \prime} \approx-\rho_{g 0} h_{m}^{0}\left(Y_{v e}-Y_{v 0}\right)
$$

which under-estimates evaporation rate compared to Eq. (7)

\section{Conclusions}

In this study, different models using Reynolds or Chilton-Colburn analogies to compute evaporation rate of a heated liquid film have been tested. The film has been supposed plane and a laminar boundary layer develops upon the film. Main conclusions are:

- Evaporation of a heated liquid film into air is accurately represented by the different models presented at low evaporation rate. Indeed, in this case, the Chilton-Colburn analogy is accurate and allows to compute $h_{m}^{0}$ from $h_{t}^{0}$ in an accurate way.

- For high evaporation rates, modeling is quite more complex. First, Reynolds and Chilton-Colburn analogies are not well established (due to the blowing velocity at the film/air interface). Secondly, the use of $C f^{0}$ and $h_{t}^{0}$ instead of $C f$ and $h_{t}$ does not take into account blowing velocity at the interface and tends to over-estimate exchanges at the film/air interface. It appears in this study that the classical model (model (6) gives good results even for high evaporation rates. This is mainly due to a compensation between several errors. Intermediate models, computed from more rigorous formulations for $\dot{m}_{v}^{\prime \prime}$ but based on analogies with $C f^{0}$ or $h_{t}^{0}$ (where the blowing velocity is neglected) give bad results at high evaporation rates.

Future works should include turbulent boundary layers with pressure gradients. At large evaporation rates, some studies $^{6}$ take into account the blowing velocity at the air/film interface. This approach is the next step of our work.

\section{References}

\footnotetext{
${ }^{1}$ Messinger, B., "Equilibrium Temperature of an Unheated Icing Surface as a Function of Air Speed," Journal of the aerospace sciences, Vol. 20, 1953, pp. 29.

${ }^{2}$ Kays, W., Crawford, M., and Weigand, B., "Convective heat and mass transfer," 1993.

${ }^{3}$ Eckert, E. and Drake Jr, R., "Analysis of heat and mass transfer," 1987.

${ }^{4}$ Spalding, D. and Spalding, D., Convective mass transfer: an introduction, McGraw-Hill, 1963.
} 
${ }^{5}$ Cousteix, J., Aérodynamique: couche limite laminaire, Éd. Cépaduès, 1988.

${ }^{6}$ Acrivos, A., "The asymptotic form of the laminar boundary-layer mass-transfer rate for large interfacial velocities," Journal of Fluid Mechanics, Vol. 12, No. 03, 1962, pp. 337-357.

\begin{tabular}{|c|c|c|}
\hline$P r$ & $S t_{t} \cdot \sqrt{R e} \cdot \mathrm{Pr}^{2 / 3}$ & relative error $(\%)$ \\
\hline 0.1 & 0.4674 & $40.8 \%$ \\
\hline 0.7 & 0.3382 & $1.86 \%$ \\
\hline 10 & 0.3354 & $1.01 \%$ \\
\hline 20 & 0.3353 & $0.987 \%$ \\
\hline
\end{tabular}

Table 1. Influence of Prandtl number $(P r)$ on Chilton-Colburn analogy.

\begin{tabular}{|c||c|c||c|c||c|c|}
\hline \multicolumn{1}{|c||}{} & \multicolumn{2}{c||}{$T_{g 0}=0^{\circ} C$} & \multicolumn{2}{c||}{$T_{g 0}=20^{\circ} C$} & \multicolumn{2}{c|}{$T_{g 0}=40^{\circ} C$} \\
\hline model & $\dot{m}_{v}^{\prime \prime} \cdot \sqrt{R e_{x}}$ & err. & $\dot{m}_{v}^{\prime \prime} \cdot \sqrt{R e_{x}}$ & err. & $\dot{m}_{v}^{\prime \prime} \cdot \sqrt{R e_{x}}$ & err. \\
\hline (1) & 0.090543907 & $0 \%$ & 0.86295014 & $0 \%$ & 3.0309404 & $0 \%$ \\
\hline (2) & 0.091657766 & $1.23 \%$ & 0.88200552 & $2.21 \%$ & 3.1840185 & $5.05 \%$ \\
\hline (3) & 0.091590629 & $1.16 \%$ & 0.87584055 & $1.49 \%$ & 3.105575 & $2.46 \%$ \\
\hline (4) & 0.091497428 & $1.05 \%$ & 0.86921656 & $0.73 \%$ & 3.0245358 & $0.21 \%$ \\
\hline (5) & 0.090935059 & $0.43 \%$ & 0.86580593 & $0.33 \%$ & 3.032169 & $0.04 \%$ \\
\hline (6) & 0.091187885 & $0.71 \%$ & 0.86654307 & $0.42 \%$ & 3.0185757 & $0.41 \%$ \\
\hline
\end{tabular}

Table 2. Comparison of different evaporation models. Reference is the model (D). Tab $1 / 2$.

\begin{tabular}{|c||c|c||c|c|}
\hline \multicolumn{1}{|l||}{} & \multicolumn{2}{c||}{$T_{g 0}=85^{\circ} C$} & \multicolumn{2}{c|}{$T_{g 0}=99.9^{\circ} C$} \\
\hline model & $\dot{m}_{v}^{\prime \prime} \cdot \sqrt{R e_{x}}$ & err. & $\dot{m}_{v}^{\prime \prime} \cdot \sqrt{R e_{x}}$ & err. \\
\hline (1) & 31.912616 & $0 \%$ & 81.369926 & $0 \%$ \\
\hline (2) & 52.629862 & $64.9 \%$ & 7316.3395 & $8891.4 \%$ \\
\hline (3) & 38.181768 & $19.6 \%$ & 298.22203 & $266.5 \%$ \\
\hline (4) & 26.768636 & $16.1 \%$ & 5.8561221 & $92.8 \%$ \\
\hline (5) & 30.319736 & $4.99 \%$ & 38.44098 & $52.76 \%$ \\
\hline (6) & 28.467332 & $10.80 \%$ & 61.748957 & $24.11 \%$ \\
\hline
\end{tabular}

Table 3. Comparison of different evaporation models. Reference is the model (1). Tab $2 / 2$. 


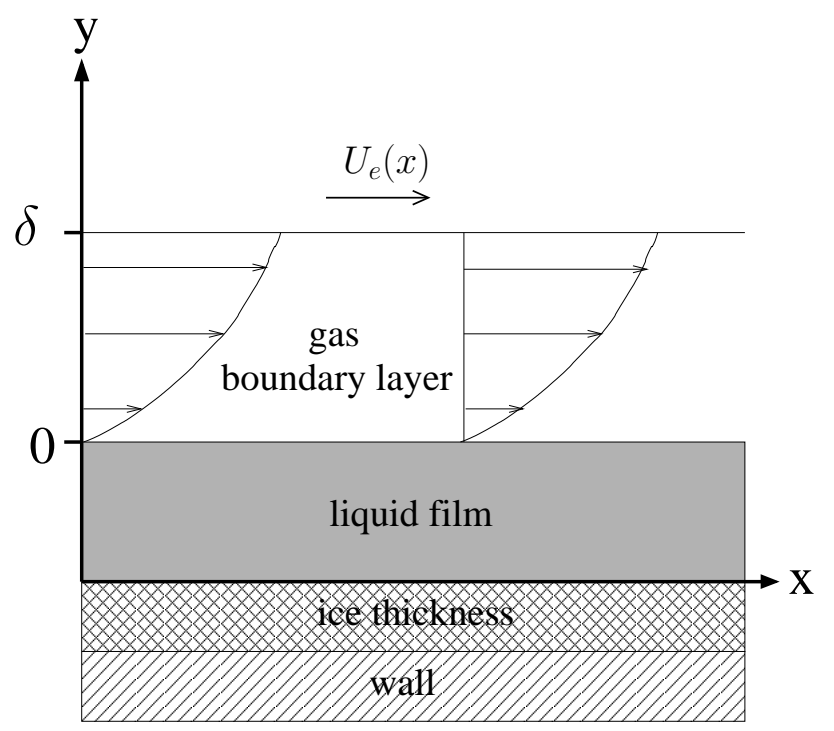

Figure 1. Scheme and notations.

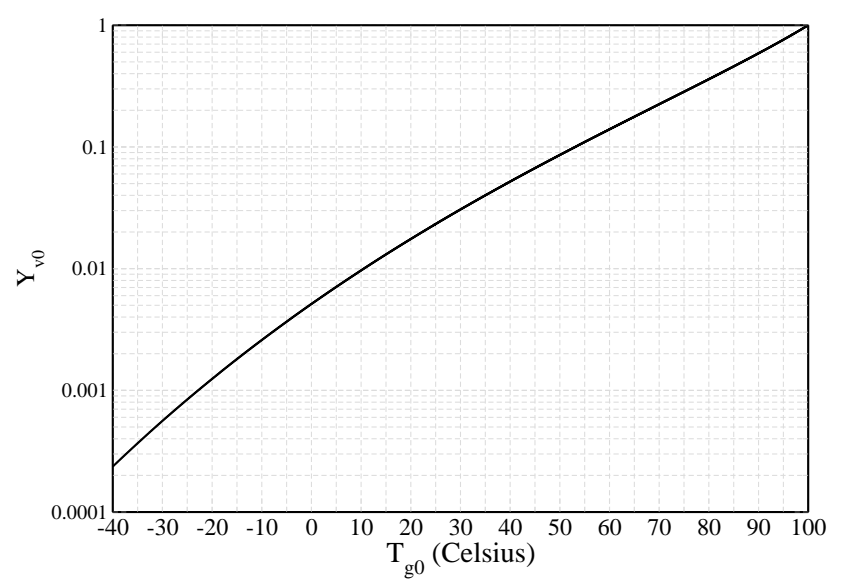

Figure 2. $Y_{v 0}$ vs. $T_{g 0}$. 

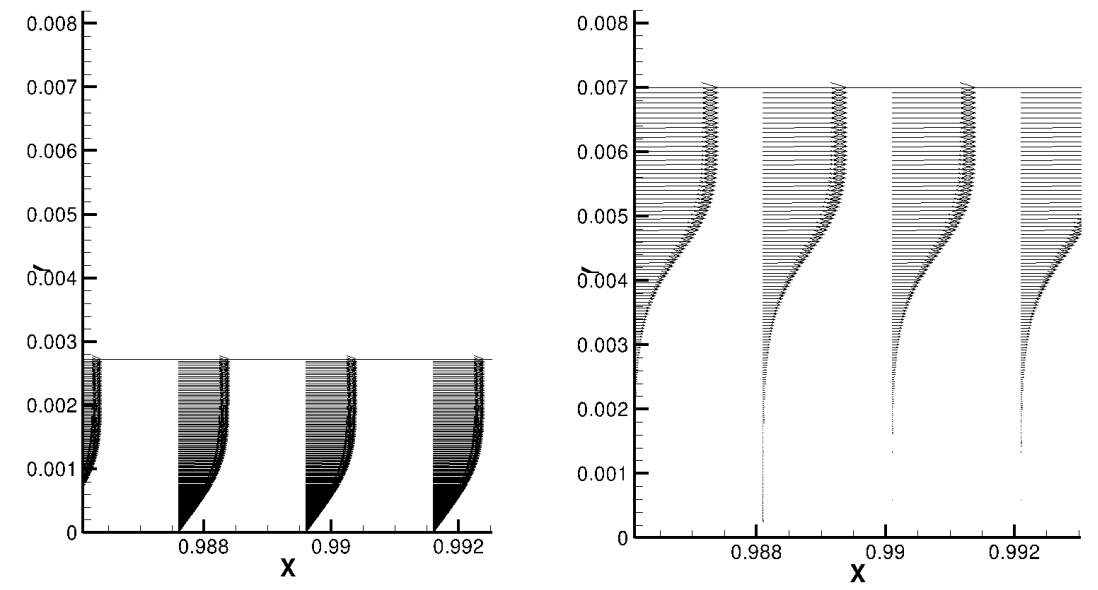

Figure 3. Influence of evaporation rate on the velocity profile inside the boundary layer. Left: $T_{g 0}=0^{\circ} C$. Right: $T_{g 0}=99.9^{\circ} \mathrm{C}$. 